Tropical Journal of Pharmaceutical Research, February 2010; 9 (1): 1-10

(C) Pharmacotherapy Group,

Faculty of Pharmacy, University of Benin

Benin City, 300001 Nigeria.

All rights reserved.

Research Article

Available online at http://www.tjpr.org

\title{
Treatment Outcomes in Patients Receiving Combination Antiretroviral Therapy in Central Hospital, Benin City, Nigeria
}

\section{Kenneth A Agu*, Uche M Ochei, Azuka C Oparah and Obialunamma U Onoh}

${ }^{1}$ Department of Clinical Pharmacy and Pharmacy Practice, University of Benin, Benin City, 300001 Nigeria; ${ }^{2}$ West African Postgraduate College of Pharmacists, Lagos, Nigeria.

\begin{abstract}
Purpose: This study investigated mortality rate, early CD4 responses, pattern of ARVs substitutions and medication adherence of HIV-infected patients on first-line triple combination antiretroviral therapy (ART) in Central Hospital, Benin City, Nigeria.

Methods: A retrospective assessment of 196 HIV-infected patients on first-line combination ART regimens was performed following 18 months of therapy. Medication adherence assessment of a 69patient follow-up target group was based on a study-specific questionnaire. Paired sample t-test and simple linear correlation were used to test the association of the CD4-cell counts at different time intervals. Kaplan-Meier model was used to assess survival functions while log-rank test was applied to assess statistical difference at $95 \%$ confidence interval (Cl). Mean age of participants was 33.6 years (95\% Cl, 32.1 - 35.2; $67.9 \%$ were females.

Results: At ART initiation, $27.0 \%$ were at WHO clinical stage II, $47.0 \%$ at stage III. Mortality rate ( $\mathrm{N}=$ 196) was 20.3 deaths per 100 patient-months; $31.6 \%$ occurred in < 30 days while $52.6 \%$ occurred post-120 days of treatment. The mean CD4-cell count $\left(\mathrm{cell} / \mathrm{s} / \mathrm{mm}^{3}\right)$ at ART initiation was 179.2 which increased to 328.5 at 3 months, 325.6 at 6 months, 357.4 at 12 months, and 366.7 at 18 months, ( $p<$ 0.01). Patients started on stavudine-based or efavirenz-based regimens were considerably more likely to have that drug substituted, compared to patients started on zidovudine-based or nevirapine-based regimens. The level of adherence reported after 18 months on ART was $73.8 \%$.

Conclusion: In this setting, patients receiving ART showed significant improvements in CD4-cell status but adherence level was relatively poor. Patients were more stable on zidovudine-based or nevirapinebased regimens than on stavudine-based or efavirenz-based regimens. Early mortality rate was high, indicating a need for early interventions.
\end{abstract}

Keywords: Antiretroviral therapy; HIVIAIDS; Mortality; Therapy outcomes, Nigeria 


\section{INTRODUCTION}

Epidemiological surveillance of HIV/AIDS in Nigeria showed an increasing prevalence from $0.000001 \%$ in 1986 to $0.22 \%$ in 1987 , $1.8 \%$ in $1991,3 \%$ in $1992,3.8 \%$ in 1993 , $4.5 \%$ in $1996,5.4 \%$ in 1999 , and $5.8 \%$ in 2001 [1]. The 2003 surveillance recorded a slight drop to $5.0 \%$; this translates to 3.8 million Nigerians living with HIV - about $20 \%$ of the HIV/AIDS burden of the African continent and $10 \%$ of the global HIV/AIDS burden. There was a further slight drop in 2005 to $4.4 \%[2,3]$. The epidemic in Nigeria can be described as heterogeneous, with various communities in different stages. Young people (age $15-24$ years) constitute a large proportion of those infected, while younger ages, primary and secondary education levels, were associated with higher risk of HIV infection with $5.9 \%$ and $5.4 \%$ prevalence, respectively $[1,4]$. The problem of HIV/AIDS in Nigeria further compounds the nation's poor health indices. The Nigerian antiretroviral therapy (ART) guideline for initiating ART in adults and adolescents is dependent on $\mathrm{WHO}$ clinical staging and availability of CD4-cell count testing [2], and endorses largely the WHO guideline on ART initiation.

In a study by Bolton-More et al, the results showed that patients starting a zidovudinebased regimen or efavirenz-based regimen were considerably more likely over time to have that drug substituted, compared with patients starting a stavudine-based regimen or nevirapine-based regimen respectively [7]. In another study at Chiradzulu District of Malawi by Ferradini et al, a simplified HAART programme was scaled up and survival indicators - CD4 count evolution, virological response, and adherence to treatment analyzed in adults who started HAART six months or more before the analysis. At follow-up (median 8.3 months), $74 \%$ were still on HAART, $19 \%$ had died, $7 \%$ were lost to follow-up, and $0.5 \%$ discontinued treatment. The study showed that WHO stage IV, male sex, and baseline CD4 count lower than 50 cells per muL were independent determinants of death in the first 6 months. At 12 months, the probability of individuals still in care was 0.76 and the median CD4 gain was 165 cells per $\mathrm{m} \mu \mathrm{L}$. Of several indicators measuring adherence, selfreported poor adherence $(<80 \%)$ in the past 4 days was the best predictor of detectable viral load [8]. Recent studies demonstrated the long-term effectiveness of antiretroviral therapy (ART) in a developing country urban setting $[9,10]$. These studies suggest the importance of monitoring ART patients more closely over the first year, even if six-month immunological and virological responses appear to be good.

The effectiveness of HIV/AIDS management depends critically on the efficacy of the antiretroviral drugs (ARVs) against the virus and adherence to medications. Recent studies indicate that triple combination ART regimens have proved efficacious in industrialised countries in response to HIV/AIDS but the prolonged effectiveness of ART in a developing countries is not well established. Furthermore, the efficacy of ARVs might not be the same for some HIV strains present in Africa [7-9]. The high cost of ART intervention, complicated procedures, inadequate infrastructures for following up patients and delivering medicines regularly, stigma and discrimination make access to care and support more difficult for HIVinfected patients. Misconceptions, cultural beliefs, poor socio-economic indices and ignorance are among the factors that could constitute the greatest challenge to wide acceptance and adherence to ART in Africa. Since adherence is the cornerstone of successful ART [11-16], there is a need to appraise the treatment outcomes of ART interventions against these factors which are considered as obstacles to mounting concerted ART programmes in Nigeria.

The evaluation of the effectiveness of combination ART regimens in the management of HIV positive patients in Nigeria is particularly important because the 
supporting efficacy data are scanty. This study aims to document the evidence of ART efficacy in Edo State, Nigeria. Edo, one of the 36 States in Nigeria, has a population of over 3.2 million and an estimated number of $>140,000$ people living with HIV. About 70,000 people in this state are in need of HIV comprehensive care services $[3,5,6]$. The objectives of this retrospective investigation were, therefore, to describe early CD4-cell response and mortality rate of HIV-infected patients on triple combination ART after 18 months, to assess the patients' pattern of ARVs substitution within the first-line ART regimens, and to assess medication adherence of those patients active on treatment after 18 months period.

\section{METHODS}

\section{Study design}

A retrospective assessment of ART patients was performed using routinely collected clinical and outcome data from the patients' hospital records. Medication adherence of patients active on treatment after 18 months of ART was prospectively assessed using the self-report adherence method.

\section{Setting}

This study was carried out in Central Hospital Benin, a secondary public health care facility in Benin City, Nigeria which offers comprehensive HIV care services, starting October 2005. We obtained administrative approval from the management of the hospital and informed consent from the study participants.

\section{Population/Sample}

The study sample was a group of 196 patients, who enrolled and commenced ART $1^{\text {st }}$ October to $31^{\text {st }}$ December, 2005. A subsample of the HIV-positive patients that were active on treatment as of October 12, 2007 was selected for a follow-up medication adherence. The sample size for this subgroup was statistically determined from the population to be 64 out of 196 and hence a target of 69 patients was followed up.

\section{Inclusion/Exclusion criteria}

All HIV positive patients who were enrolled and commenced ART within the study period, and refilled their prescriptions at the Pharmacy Department of the facility were included. Only the patients indicated above who were active on treatment as at October 12, 2007 were included for medication adherence assessment. Participants consented to their inclusion. All those patients who did not meet the required criteria or give informed consent were excluded from the study.

\section{Data collection}

An unobtrusive technique was used to collect relevant data from the manually maintained records of the 196 participants using a structured instrument. Twenty patients were used in the pilot study for this aspect of the investigation. A study-specific questionnaire was administered to the sub-sample of 69 patients. This was done over a period of three and half months as this sub-sample had varying medication refill appointment dates. Similarly, 5 patients were involved in the pilot study prior to the main data collection. A researcher and two trained assistants administered the survey instruments.

\section{Data analysis}

The data were analyzed using SPSS version 10. Paired sample $t$-test and simple linear correlation were used to test the association of the CD4-cell counts at different time intervals. Kaplan-Meier model was used to assess survival functions stratified by baseline CD4-cell count and WHO clinical stage, and the log-rank test was used to assess statistical difference among groups (for equality of survival distributions). Mortality rates overall, at $<30$ days, $30-$ 60days, $61-120$ days and $>120$ days were 
determined using the Life Table. All reported $\mathrm{P}$-values were 2 -sided, and $\mathrm{P}<0.05$ was used to determine statistical significance.

\section{RESULTS}

\section{Socio-demographic characteristics of patients}

Of the 196 study participants, 194 (99.0\%) were treatment-naïve while $2(1.0 \%)$ were already receiving ART prior to enrollment. The mean age of the 196 patients at initiation of antiretroviral treatment was 33.6 years (95 $\% \mathrm{Cl}, 32.1$ - 35.2). Other socio-demographic characteristics of the patients are presented in Table 1.

\section{Patients' baseline clinical characteristics}

Baseline weights were available for 178 patients $(90.8 \%)$ with a mean value of 56.6 $\mathrm{kg}$ while $18(9.2 \%)$ had no documented baseline weights; the median CD4-cell count at initiation of antiretroviral treatment was 101 (IQR, 35 - 206) cells $/ \mathrm{mm}^{3}$. Those that were ART-eligible and initiated treatment based exclusively on the CD4-cell count result was $142(72.4 \%)$, while a few $(21.4 \%)$ were based exclusively on the clinical staging. Pretreatment medication adherence counselling was given to 193 (98.5\%) patients while 3 $(1.5 \%)$ had no documentation of this service (Table 2).

\section{Experience with first-line antiretroviral drug regimens}

The 196 patients were started on antiretroviral first-line drug regimen and remained on it until the last medication refill visit in the Pharmacy records. Of 196 patients initiating ART, $40.8 \%$ were placed on stavudine+lamivudine+nevirapine, $46.9 \%$ on zidovudine+lamivudine+ nevirapine, $7.7 \%$ on zidovudine +lamivudine+efavirenz, while 4.6 $\%$ were placed on stavudine+ lamivudine+ efavirenz. There were no switches from firstline regimens to second-line or salvage regimens.
Overall, $14.8 \%$ patients had single-drug substitution of their nucleoside reverse transcriptase inhibitors, $0.5 \%$ had a two-drug substitution of nucleoside reverse transcriptase inhibitor and non-nucleoside reverse transcriptase inhibitor (i.e., "stavudine+nevirapine" substituted to "tenofovir+efavirenz") while $5.1 \%$ had singledrug substitutions of their non-nucleoside reverse transcriptase inhibitors. These substitutions were due to few cases of drug intolerance, toxicity, or pregnancy, and many cases of undocumented reasons. Of $45.4 \%$ patients initiated with stavudine-based regimen, $15.7 \%$ switched from stavudine to zidovudine. Additionally, $13.1 \%$ of $54.6 \%$ patients initiated with zidovudine-based regimen switched from zidovudine to stavudine. Out of $87.8 \%$ patients receiving a nevirapine-based regimen, $3.5 \%$ switched to efavirenz (of which $33.3 \%$ switched back to nevirapine), while of $12.2 \%$ patients starting an efavirenz-based regimen, $3.5 \%$ switched to nevirapine.

\section{Survival outcomes}

As of October 12, 2007, out of the total 196 patients, $19.4 \%$ were documented to have died over 187 patient-months of follow-up (mortality rate of 20.3 deaths per 100 patientmonths); $38.8 \%$ were active and still on treatment at the facility, $6.6 \%$ transferred out to other facilities; $3.0 \%$ stopped treatment on medical advice, of which $83.3 \%$ were due to pregnancy and $16.7 \%$ documented to have gone on drug holiday based on medical advice due to high CD4-cell count. A total of $27.6 \%$ of patients could not be followed up due to logistic reasons while $4.6 \%$ patients' hospital case notes were missing and could not be traced.

\section{Mortality in ART patients}

The actual causes of death in these cases were not documented. Of the 38 dead patients having a baseline mean weight of $49.1 \mathrm{Kg}$ and mean age of 36.0 years, the 
Table 1: Socio-demographic characteristics of patients

\begin{tabular}{|c|c|c|}
\hline Characteristics & Frequency $(\mathrm{N}=196)$ & $\%$ \\
\hline \multicolumn{3}{|l|}{ SEX } \\
\hline Male & 63 & 32.1 \\
\hline Female & 133 & 67.9 \\
\hline \multicolumn{3}{|l|}{ AGE } \\
\hline$\leq 15$ & 9 & 4.6 \\
\hline $16-25$ & 25 & 12.8 \\
\hline $26-35$ & 79 & 40.3 \\
\hline $36-45$ & 62 & 31.6 \\
\hline $46-55$ & 16 & 8.2 \\
\hline$\geq 56$ & 5 & 2.5 \\
\hline \multicolumn{3}{|l|}{ MARITAL STATUS } \\
\hline Single & 60 & 30.6 \\
\hline Married & 104 & 53.1 \\
\hline Widow(er) & 22 & 11.2 \\
\hline \multirow{2}{*}{\multicolumn{3}{|c|}{ EDUCATIONAL STATUS }} \\
\hline & & \\
\hline None & 11 & 5.6 \\
\hline Primary & 69 & 35.2 \\
\hline Secondary & 70 & 35.7 \\
\hline Post Secondary & 31 & 15.8 \\
\hline Not indicated & 15 & 7.7 \\
\hline \multicolumn{3}{|l|}{ OCCUPATIONAL STATUS } \\
\hline Unemployed & 72 & 36.7 \\
\hline Employed & 25 & 12.8 \\
\hline Self-employed & 49 & 25.0 \\
\hline Not indicated & 44 & 22.4 \\
\hline Retired & 1 & 0.5 \\
\hline Student & 5 & 2.6 \\
\hline
\end{tabular}

Table 2: Baseline clinical characteristics

\begin{tabular}{ccc}
\hline Characteristics & Frequency $(\mathbf{N}=\mathbf{1 9 6})$ & $\%$ \\
\hline WHO Clinical Stage & 42 & 21.4 \\
I & 53 & 27.0 \\
III & 92 & 47.0 \\
IV & 6 & 3.1 \\
Not indicated & 3 & 1.5 \\
CD4-cells Count (cells $\left./ \mathbf{m m}^{3}\right)$ & & \\
$<10$ & 21 & 10.7 \\
$10-49$ & 44 & 22.4 \\
$50-100$ & 31 & 15.8 \\
$101-200$ & 46 & 23.5 \\
$201-350$ & 25 & 12.8 \\
$>350$ & 26 & 13.3 \\
Not indicated & 3 & 1.5 \\
Working & & \\
Ambulatory & 147 & 75.0 \\
Bed-ridden & 35 & 17.8 \\
Not indicated & 5 & 2.6 \\
\end{tabular}


Table 3: Mortality in ART patients

\begin{tabular}{ccccc}
\hline Time interval & $\begin{array}{c}\text { Number } \\
\text { entering } \\
\text { this interval }\end{array}$ & Deaths & $\begin{array}{c}\text { Follow-up } \\
\text { (patient- } \\
\text { months) }\end{array}$ & $\begin{array}{c}\text { Mortality rate } \\
\text { (deaths per 100 } \\
\text { patient-months) } \pm \text { SD }\end{array}$ \\
\hline Overall & 196 & 38 & 187 & $20.32 \pm 0.66$ \\
$<30$ days & 187 & 12 & 168 & $7.14 \pm 0.51$ \\
$30-60$ days & 137 & 4 & 133 & $3.01 \pm 0.06$ \\
$61-120$ days & 125 & 2 & 123.5 & $1.62 \pm 0.01$ \\
$>120$ days & 115 & 20 & 113.5 & $17.62 \pm 0.11$ \\
\hline
\end{tabular}

baseline median CD4-cell count at initiation of antiretroviral treatment was 44 (IQR, 7 to 85) cells $/ \mathrm{mm}^{3}$. $2.6 \%$ had no documented baseline CD4-cell count but presented at stage IV. Of the dead cases, $81.6 \%$ presented for treatment at either stage II or III, while those that presented at WHO clinical stage III, $63.2 \%$, were more than 3-fold higher than those that presented at $\mathrm{WHO}$ clinical stage II (18.4\%); $7.9 \%$ were stage I and $10.5 \%$ were stage IV. In terms of functional status, $63.2 \%$ were either ambulatory (55.3 \%) or bedridden (7.9 \%) at the time of presentation for treatment while $36.8 \%$ were working.

The mean treatment duration before death was found to be $282.5(95 \% \mathrm{Cl}, 243.2$ to 321.9) days while the mortality rate was 20.32 deaths per 100 patient-months (Table $3)$.

The mean survival times before death for the ART patients with a baseline CD4-cell Count of $<50$ and $50-100$ cells $/ \mathrm{mm}^{3}$ were least for baseline stage IV, being 528.5 and 351.0 days, respectively, but there was no significant difference $(p>0.05)$ for both CD4cell count categories for baseline stages I IV $\left(<50\right.$ cells $/ \mathrm{mm}^{3}, \mathrm{p}=0.3866$; and $50-$ 100 cells $/ \mathrm{mm}^{3}, p=0.4578$ ). Considering the baseline functional status, the mean survival times before death for the ART patients working and ambulatory were also least for baseline stage IV, yielding 56.0 and 434.0 days respectively, but there was a significant difference $(p<0.05)$ for the category working at baseline $\mathrm{WHO}$ clinical stage I - IV $(\mathrm{p}=$ 0.0296) while that of ambulatory patients was not significantly different for baseline WHO clinical stage I - IV $(p=0.1710)$.

\section{Loss to follow-up}

Of the 54 patients lost to follow-up, their baseline mean weight was $56.2 \mathrm{~kg}$ and mean age was 30.8 (IQR, 25 - 37) years. The median CD4-cell Count at initiation of ART was 142.5 (IQR, $50.5-296.0$ ) cells $/ \mathrm{mm}^{3}$. Of these patients, $61.1 \%$ had baseline CD4-cell Count > 100 cells $/ \mathrm{mm}^{3}$ while $38.9 \%$ had baseline CD4-cell Count of $\leq 100 \mathrm{cells} / \mathrm{mm}^{3}$ of which $25.9 \%$ were $<50$ cells $/ \mathrm{mm}^{3}$. That compared considerably better to the baseline parameters for the death cases reported above. Those presented for treatment at either stage I $(33.3 \%)$ or stage II $(27.8 \%)$ were $61.1 \%$ while 35.2 and $3.7 \%$ were at stages III and IV, respectively. For functional status, $90.7 \%$ was reported to be working while $5.6 \%$ were ambulatory and $3.7 \%$ were bedridden. Of the 147 patients that had a working functional status, loss to follow-up patients contributed $33.3 \%$.

The mean duration of treatment before loss to follow-up was $392.3(95 \% \mathrm{Cl}, 350.8$ to 433.7) days. Of 54 patients lost to follow-up, $24.1 \%$ were lost to follow-up in $<30$ days of treatment, $7.4 \%$ in 30 - 90 days, $1.8 \%$ in 91 - 
180 days and $66.7 \%$ post- 180 days of treatment.

\section{Patients active on ART at the end of $\mathbf{1 8}$ months}

Of the 76 patients still on treatment, the baseline mean weight was $59.5 \mathrm{~kg}$ with a mean age of 34.4 (IQR, 29 to 39.25) years and the median pre-treatment CD4-cell Count of 113 (IQR, 37-190) cells $/ \mathrm{mm}^{3}$. Of these, $52.6 \%$ had baseline CD4-cell Count $>100$ cells $/ \mathrm{mm}^{3}$ while $47.4 \%$ had baseline CD4cell Count of $\leq 100$ cells $/ \mathrm{mm}^{3}$ of which $34.2 \%$ were $<50$ cells $/ \mathrm{mm}^{3}$. All the patients received pre-treatment medication adherence counselling. The pre-treatment WHO Clinical staging of this category of patients comprised $15.8 \%$ at stage I, $34.2 \%$ at stage II, $50.0 \%$ at stage III, and none was at stage IV while the baseline functional status was $86.8 \%$ working, $13.2 \%$ ambulatory, and none was bedridden.

\section{CD4-cell Count changes over time}

The CD4-cell response analysis was limited to 186 patients who had a baseline CD4 measurement. The mean CD4-cell count at ART initiation was $179.2(95 \% \mathrm{Cl}, 143.6$ 220.9) cells $/ \mathrm{mm}^{3}$ and increased to 328.5 (95 $\% \mathrm{Cl}, 294.7-362.3)$ cells $/ \mathrm{mm}^{3}$ at 3 months (80.3\% increase from the mean baseline value) among the 75 of 135 (55.6\%) active patients who had been enrolled long enough to have a 3-month measurement. This increased to $325.6(95 \% \mathrm{Cl}, 290.5$ - 360.6) cells $/ \mathrm{mm}^{3}$ at 6 months $(78.7 \%$ increase from the mean baseline value) among the 62 of $118(52.5 \%)$ active patients enrolled long enough to have a 6-month measurement. At 12 months, it increased further to 357.4 (95 $\% \mathrm{Cl}, 320.4$ - 394.3) cells $/ \mathrm{mm}^{3}$ (96.1\% increase from the mean baseline value) among the 55 - $98(56.1 \%)$ active patients enrolled long enough to have a 12-month measurement, and finally to $366.7(95 \% \mathrm{Cl}$, 328.3 - 405.1) cells $/ \mathrm{mm}^{3}$ at 18 months (101.2 $\%$ increase from the mean baseline value) among the 66 of $76(86.8 \%)$ active patients enrolled long enough to have an 18-month measurement. The percent increase from the patients' baseline CD4-cell Counts (cells $/ \mathrm{mm}^{3}$ ) at the different time intervals was significant at both 0.01 and 0.05 probability levels $(p<0.01)$ but there was no statistical difference between the following pairs of CD4-cell Count measurements - at $3^{\text {rd }}$ and $6^{\text {th }}$ month, $6^{\text {th }}$ and $12^{\text {th }}$ month, $12^{\text {th }}$ and $18^{\text {th }}$ month $(p>0.05)$. The correlation of the CD4cell Counts measured at the different time intervals was found to be positive and statistically significant at 0.05 probability level.

\section{Medication adherence}

Of the 69 patients $(90.8 \%)$ that were reached, out of those 76 patients still on treatment, there were $65.2 \%$ females and $34.8 \%$ males. The age distribution of the 69 patients reached at the time of assessment showed that a majority of them $(68.1 \%)$ were between the ages of 26 and 45 years, $18.9 \%$ patients were above 45 years while $13.0 \%$ were below 25 years. A majority of the patients $(55.1 \%)$ were married, $26.1 \%$ were single while $17.4 \%$ and $1.4 \%$ of the patients were widowed and either divorced or separated, respectively. Only $17.4 \%$ had post-secondary education while $79.7 \%$ had either secondary school $(42.0 \%)$ or primary school education (37.7 \%). The greater percentage of patients $(79.7 \%)$ were employed, including those that were selfemployed while only a small proportion were unemployed $(11.6 \%)$ and $7.2 \%$ were students.

Of the 69 patients, $89.9 \%$ reported knowing the benefits of antiretroviral therapy (of which almost all respondents, $98.4 \%$ got it rightly stated) while $10.1 \%$ reported not knowing the benefits of ART. A majority of the respondents, $84.1 \%$, had a good knowledge of the importance of medication adherence; a few, $14.5 \%$, did not, while $1.4 \%$ was not sure of this. The respondents reported a good therapeutic relationship with the 
healthcare providers with a mean score of $88.4 \%$.

\begin{abstract}
All the 69 respondents reported receiving medication adherence counseling before starting ART. On following the scheduled specific timing for taking the medications, the respondents had a mean score of $64.1 \%$ while the mean score for taking the right drug in the right dose at the right frequency, irrespective of the specific timing, was 83.5 $\%$. Overall, the 69 respondents recorded 73.8 $\%$ level of adherence to medications.
\end{abstract}

The patients that reported not missing any dose of their medications were $35(50.7 \%)$ while $34(49.3 \%)$ had missed one or more pills (of which 30 , or $88.2 \%$ of them, missed 6 doses and less) in the previous two months. The occupation of the respondents was associated with adherence $(p=0.00142)$ while educational status of the respondents was not $(p=0.0971)$.

The three most reported reasons for missed doses were: busy working or at school (41.9 $\%$ ), patient moved away from home or travelled (19.8\%), and forgetfulness (14.0 $\%$ ). Of the 69 respondents, $59.4 \%$ were taking one to three pills per day while $36.2 \%$ were taking at least five pills per day.

\section{DISCUSSION}

Analysis of the demographic profile of the patients showed that a majority of those most at risk of HIV infection were youths and the proportion of females in the treatment group was more than two-fold greater than that of the males. This is consistent with a recent study [3]. Almost all ART initiation among the participants was done in line with the provision of the Nigeria ART guidelines [2].

A large proportion of the patients presented very late for treatment with very poor baseline parameters such as CD4-cell count and WHO Clinical stage; for example, half presented for treatment with WHO Clinical stage of either III or IV. This finding supports the need for a rapid scale-up of counselling and testing for early detection of asymptomatic cases in developing countries. The study finding is also consistent with the results of Bolton-more et al, that patients starting a zidovudine-based regimen or efavirenz-based regimen were considerably more likely over time to have that drug substituted, compared with patients starting a stavudine-based regimen or nevirapinebased regimen respectively [7]. The participants were stable on the first-line regimen and none of the patients switched to the $2^{\text {nd }}$-line or $3^{\text {rd }}$-line antiretroviral regimens.

The proportion of the participants still on treatment at the end of 18 months period was relatively low compared to the study findings of Ferradini et al [8] while the number of fatal cases was similar but loss to follow-up was higher. Mortality rate was high probably due to poor baseline parameters or late presentation for treatment. The findings showed that a considerably high percentage of patients with good CD4-cell response presented relatively early for treatment at a relatively higher baseline CD4-cell Count, stage I or II and better functional status, compared to the reported dead cases. Therefore, relatively high baseline parameters with good adherence could be considered as good prognostic factors.

In this study, patients receiving ART showed significant improvements in CD4-cells status at $3,6,12$ and 18 months of antiretroviral therapy. This is consistent with recent studies [8-10]. Therefore, the early immunological response of HIV-infected patients on ART after a period of 18 months was considerably good in our setting. But mortality within the first 120 days of starting therapy was high. Although many patients were ill at presentation, those who survived past the first 120 days of therapy generally had good outcomes thereafter, a phenomenon that has been observed among adults in other developing-world settings [7]. 
As has been described in other regions [7], the result showed that surviving patients generally have very good CD4-cell responses. The average patient in the cohort experienced a more than doubling of his or her CD4-cell count in the first 18 months of ART. It was also observed that follow-up CD4-cells measurements were not done every 6 months for all patients eligible for repeat CD4-cell Count in accordance to the national ART guidelines [2]. On the average, these follow-up CD4-cells measurements were repeated in about two-third of the cases, hence the need for improvement in the adherence to the national treatment guideline [2].

Adherence to medication and lifestyle changes is a key factor to positive treatment outcome in the therapy of HIV/AIDS and this presents a challenge to both patients and healthcare providers [11-16]. The patients' knowledge of the benefits of ART and consequences of non-adherence of a majority was found to be high and that might have enhanced medication adherence and possibly the survival of patients on ART. From the result in this treatment centre, it can be deduced that these patients were well educated and counselled on the basic facts about ART before or after treatment initiation. The respondents also reported a good therapeutic relationship with the healthcare providers which are considered very essential for a successful adherence to medications. Despite these and the fact that all the respondents were counseled on medication adherence before starting ART, adherence was still found to be poor, $73.8 \%$, compared to the $100 \%$ recommended level of adherence required to achieve the goal of ART. This is consistent with a recent study of self-reported poor adherence (< 80\%) [8]. With this level of adherence, good CD4 responses were obtained which may be better with increasing level of adherence.

The educational status of the participants was not found to be associated with adherence as their occupational status. This was consistent with the major reasons reported by the respondents for nonadherence (busy working/at school). It was very remarkable to observe that pill burden, cost, out of stock of medicines at the dispensary and lack of understanding of how to take drugs, etc, were never given as reasons for non-adherence to medications by the respondents. Despite all these, immunological response was good.

\section{LIMITATIONS}

The researchers were unable to create a control group and hence treatment outcome for every patient was assessed by comparing the pre-ART CD4-cells counts and CD4-cells counts while on ART. Fluctuations in absolute CD4 count are known to occur and hence definite conclusions should be drawn from repeated measurements [17] but that was not the case with this study. The duration of treatment was calculated as the time difference between the date of first ART initiation and the last date of medication refill at the ART pharmacy. Patients' last date of medication refill at the pharmacy was used as the date of death. The exact date of death could not be obtained since almost all death cases took place outside the hospital setting and was determined through contact tracing and home visits. The actual causes of the death could also not be ascertained. There were cases of missing or incomplete records that hampered the study. Some aspects of data collection were based on self-report, which could be biased.

\section{CONCLUSION}

In this setting, patients receiving ART showed significant improvements in CD4-cell status and early CD4-cell response but adherence level was relatively poor. Patients were more stable on zidovudine-based or nevirapinebased regimens compared to stavudinebased or efavirenz-based regimens. Early mortality rate was high, indicating a need for early interventions. 


\section{CONFLICT OF INTEREST}

We declare no conflicts of interest.

\section{REFERENCES}

1. Federal Ministry of Health, FMOH. Technical Report, National HIV/Syphilis Sero-prevalence Sentinel Survey among Pregnant Women Attending Antenatal Clinics in Nigeria, 2005; 57.

2. Federal Ministry of Health, FMOH. Guidelines for the use of Antiretroviral (ARV) drugs in Nigeria. 2005; pp 25-47.

3. Ngwai YB, Odama LE. HIVIAIDS Prevention Services In: Skill Certification Workshop on HIVIAIDS, STIs, and Opportunistic Infections for Community Pharmacists, Participant's Manual June 2006; 5-6.

4. GHAINing Ground. A Newsletter from Global HIV/AIDS Initiative Nigeria, Family Health International, 2005; 1 - 4.

5. GHAINing Ground. A Newsletter from Global HIVIAIDS Initiative Nigeria, Family Health International. 2006; $2-5$.

6. Family Health International. Update on Global HIVIAIDS initiative Nigeria (GHAIN), Focus on Edo state, Institute for HIVIAIDS, Arlington, USA. December 2006; 1-4.

7. Bolton-Moore C, Mubiana-Mbewe M, Cantrell RA, Chintu N, Stringer EM, Chi BH. Clinical Outcomes and CD4-cell Response in Children Receiving Antiretroviral Therapy at Primary Health Care Facilities in Zambia. JAMA 2007; 298(16): 1888-1899.

8. Ferradini L, Jeannin A, Pinoges L, Izopet J, Odhiam bo $D$, Mankhambo L. Scaling up of highly active antiretroviral therapy in a rural district of Malawi: an effectiveness assessment, Lancet 2006; 367(9519): 1335 - 1342.
9. Corey $D M$,

Kim HW, Salazar R, Illescas R, Villena J, Gutie rrez L, Sanchez J, Tabet SR. Brief report: effectiveness of combination antiretroviral therapy on survival and opportunistic infections in a developing world setting: an observational cohort study. The Journal of Acquired Immune Deficiency Syndromes, 2007; 44(4): 451- 455.

10. Sabin CA (2005). Baseline Characteristics Influence Benefits from Highly Active Antiretroviral Therapy. AIDS Journal, 2005; 19: pp 19952000.

11. Osterberg L, Blaschke T. Adherence to medication. N Engl J Med 2005; 353: 487-497.

12. Praska JL, Kripalani S, Seright AL, Jacobsen TA. Identifying and assisting low-literacy patients with medication use: a survey of community pharmacies. Ann Pharmacother. 2005; 39: 1441-1445.

13. Vanhove GF, Schapiro JM, Winters MA. Patient compliance and drug failure in protease inhibitor monotherapy. JAMA 1996; 276: 19551956.

14. Deeks S, Beatty G, Cohen PT. Viral load and CD4+ $T$-cell changes in patients failing potent protease inhibitor therapy. In: Program and abstracts of the 5th Conference on Retroviruses and Opportunistic Infections; February 1-5, 1998; Chicago. Abstract 419.

15. Montaner JS, Reiss P, Cooper D. A randomized, double-blind trial comparing combinations of nevirapine, didanosine, and zidovudine for HIV-infected patients: the INCAS Trial. Italy, the Netherlands, Canada, and Australia Study. JAMA 1998; 279: 930-937.

16. Melbourne KM., Geletko SM, Brown SL, WilleyLessne C, Chase S, Fisher A. Medication Adherence in Patients with HIV Infection: $A$ Comparison of Two Measurement Methods AIDS Journal. 1999; Read 9(5): 329 - 338.

17. Palella FJ, Deloria-Knoll M, Chmiel JS. Survival benefit of initiating antiretroviral therapy in HIVinfected persons in different CD4+ cell strata. Ann Intern Med 2003; 138(8): 620 - 626. 